\title{
The peripheral monocyte count is associated with the density of tumor- associated macrophages in the tumor microenvironment of colorectal cancer: a retrospective study
}

\author{
Masatsune Shibutani, Kiyoshi Maeda, Hisashi Nagahara, Tatsunari Fukuoka, Shigetomi Nakao, Shinji Matsutani, \\ Kosei Hirakawa and Masaichi Ohira
}

\begin{abstract}
Background: Inflammation is widely recognized to play an important role in cancer progression, and the peripheral monocyte count has been reported to correlate with the prognosis in patients with colorectal cancer. This is based on the hypothesis that the peripheral monocyte level and the density of tumor-associated macrophages (TAMs) in the cancer microenvironment correlate with each other. However, the influence of TAMs on the prognosis and the correlation between the peripheral monocyte count and the density of TAMs have not yet been elucidated.

Methods: A total of 168 patients with stage II/III colorectal cancer were enrolled in this study. Preoperative blood samples were obtained at the time of the diagnosis before surgery. The expression of TAMs in the cancer microenvironment was assessed by immunohistochemistry.

Results: The progression-free and overall survival rate were significantly worse in the high-TAMs group than in the low-TAMs group ( $p=0.0012$ and $p=0.0207$, respectively). The peripheral monocyte count was significantly associated with the number of TAMs (correlation coefficients: $0.202, p=0.047$ ).

Conclusions: The peripheral monocyte count was associated with the density of the TAMs, which created a microenvironment favorable for cancer development and were correlated with a poor prognosis. Therefore, the peripheral monocyte count is a useful prognostic marker reflecting the status of the tumor microenvironment.
\end{abstract}

Keywords: Colorectal cancer, Monocyte, Tumor-associated macrophage

\section{Background}

Inflammation is widely recognized to play an important role in cancer progression [1], and various inflammatory markers have been reported as useful prognostic markers in patients with various types of cancer [2-6]. The peripheral monocyte count, which is one such inflammatory marker, has been reported to correlate with the prognosis in patients with prostate, breast and

\footnotetext{
* Correspondence: fbxbj429@ybb.ne.jp

Department of Surgical Oncology, Osaka City University Graduate School of Medicine, 1-4-3 Asahi-machi, Abeno-ku, Osaka City, Osaka Prefecture 545-8585, Japan
}

colorectal cancer [7-9]. Moreover, in our previous study, the same results were obtained in colorectal cancer [10]. Tumor-associated macrophages (TAMs) are macrophages that exist within the tumor microenvironment and are derived from circulating monocytes [11, 12]. There are two kinds of macrophage phenotypes: the M1 phenotype has antitumor activity, whereas the M2 phenotype promotes cancer progression [13, 14]. Most TAMs have an M2-like phenotype and promote metastasis, angiogenesis, and immunosuppression [15].

The concept of the peripheral monocyte count being a useful prognostic marker in cancer patients is based on the hypothesis that the peripheral monocyte count 
reflects the density of TAMs in the cancer microenvironment $[9,10,16]$. However, the influence of TAMs on the prognosis and the correlation between the peripheral monocyte count and the density of TAMs have not been elucidated.

In this study, we evaluated the prognostic significance of TAMs and clarified the correlation between the peripheral monocyte count and the density of TAMs in patients with colorectal cancer.

\section{Methods \\ Patients}

A total of 168 patients with stage II/III colorectal cancer were enrolled in this study. All patients underwent potentially curative surgery for colorectal cancer at the Department of Surgical Oncology of Osaka City University between 2007 and 2009. Patients who received preoperative therapy, underwent emergency surgery for perforation/obstruction, or who had inflammatory bowel disease were excluded from this study.

The patient characteristics are listed in Table 1. A total of 85 males and 83 females were included in this study. The median age of the patients at the initial surgery was 67 years old (range: 26 to 90 years old). Ninety patients had primary tumors located in the colon, and 78 had primary tumors located in the rectum. The resected specimens were pathologically classified according to the seventh edition of the UICC TNM classification of malignant tumors [17]. The distribution of cancer stages was as follows: stage II, 92 patients; stage III, 76 patients. All patients were followed up regularly with physical and blood examinations, including measurements of the levels of tumor markers, such as carcinoembryonic antigen (CEA) and carbohydrate antigen 19-9 (CA19-9), and mandatory screening using colonoscopy and computed tomography until December 2016 or death.

\section{Blood sample analysis}

Preoperative blood samples were obtained at the time of the diagnosis before surgery. The differential white blood cell count was analyzed using an XE-5000 hematology analyzer (Sysmex, Kobe, Japan) in accordance with the manufacturer's protocol.

\section{Immunohistochemistry}

CD163 has been used as a specific marker to identify M2 macrophages $[13,14]$. Surgically resected specimens were retrieved to perform immunohistochemistry. Sections $4 \mu \mathrm{m}$ in thickness were deparaffined and rehydrated. The sections were then subjected to endogenous peroxidase blocking in $1 \% \mathrm{H}_{2} \mathrm{O}_{2}$ solution in methanol for $15 \mathrm{~min}$. Antigen retrieval was performed by autoclaving the
Table 1 Patient characteristics

\begin{tabular}{|c|c|}
\hline \multicolumn{2}{|l|}{ Gender } \\
\hline Male & 85 \\
\hline Female & 83 \\
\hline \multicolumn{2}{|l|}{ Age (years) } \\
\hline Median (range) & $67(26-90)$ \\
\hline \multicolumn{2}{|c|}{ Location of primary tumor } \\
\hline Colon & 90 \\
\hline Rectum & 78 \\
\hline \multicolumn{2}{|l|}{ Tumor depth ${ }^{a}$} \\
\hline $\mathrm{T} 1-3$ & 109 \\
\hline $\mathrm{T} 4$ & 59 \\
\hline \multicolumn{2}{|l|}{ Tumor diameter (cm) } \\
\hline Median (range) & $5.0(1.0-11.0)$ \\
\hline \multicolumn{2}{|l|}{ Histological type } \\
\hline Well, Moderately & 154 \\
\hline Poorly, Mucinous & 14 \\
\hline \multicolumn{2}{|c|}{ Lymphatic involvement } \\
\hline Negative & 40 \\
\hline Positive & 128 \\
\hline \multicolumn{2}{|l|}{ Venous involvement } \\
\hline Negative & 137 \\
\hline Positive & 31 \\
\hline \multicolumn{2}{|c|}{ Lymph node metastases } \\
\hline Negative & 92 \\
\hline Positive & 76 \\
\hline \multicolumn{2}{|c|}{ Peripheral monocyte count (/mm3) } \\
\hline Median (range) & $348(28-719)$ \\
\hline \multicolumn{2}{|c|}{ The number of TAMs (/field) } \\
\hline Median (range) & $7.67(0.67-58.67)$ \\
\hline
\end{tabular}

sections at $105{ }^{\circ} \mathrm{C}$ for $10 \mathrm{~min}$ in Dako Target Retrieval Solution (Dako, Glostrup, Denmark). Serum blocking was performed with $10 \%$ normal rabbit serum for $10 \mathrm{~min}$. After $\mathrm{H}_{2} \mathrm{O}_{2}$ and serum blocking, the slides were incubated with primary mouse monoclonal anti-CD163 antibody (1:200 dilution; Leica Biosystems, Newcastle Upon Tyne, UK) at room temperature for $1 \mathrm{~h}$. The secondary antibody was biotin-labeled rabbit anti-mouse IgG (1:500; Nichirei, Tokyo, Japan). Detection was performed with a DAB kit (Histofine simple stain kit; Nichirei). The sections were counterstained with hematoxylin.

\section{Immunohistochemical evaluations}

Immunohistochemical evaluations were carried out by two independent pathologists blinded to the clinical 
information. The number of immunoreactive macrophages at the invasive margin was counted with a light microscope in a randomly selected field at a magnification of $400 \times$ (Fig. 1). The mean of the values obtained in five different areas was used for the data analysis. According to the median TAM value, we set 8.0 as the cut-off value for the evaluation of TAMs and classified patients into a high-TAMs group and a low-TAMs group.

\section{Statistical analyses}

The significance of the correlations between TAMs and the clinicopathological characteristics were analyzed using the $X^{2}$ test and Fisher's exact test. The duration of the survival was calculated using the Kaplan-Meier method. Differences in the survival curves were assessed using the log-rank test. A multivariate analysis was performed using the Cox proportional hazard model. Associations between peripheral monocyte count and the density of TAMs in the tumor microenvironment were evaluated by Spearman's rank correlation coefficient. All of the statistical analyses were conducted using the SPSS software package for Windows (SPSS Japan, Tokyo, Japan). $P$ values of $<0.05$ were considered to indicate statistical significance.

\section{Ethical considerations}

This research conformed to the provisions of the Declaration of Helsinki. All patients were informed of the investigational nature of this study and provided their written informed consent. This retrospective study was approved by the ethics committee of Osaka City University (approved No.926).

\section{Results}

Correlations between the density of TAMs and the clinicopathological factors

The density of TAMs showed no significant relationship with any of the clinicopathological parameters, except for the histological type (Table 2).

\section{Survival analyses according to the density of TAMs}

The progression-free survival rate was significantly worse in the high-TAMs group than in the low-TAMs group ( $p=0.0012)$ (Fig. 2). The overall survival rate was also significantly worse in the high-TAMs group than in the low-TAMs group ( $p=0.0207$ ) (Fig. 3).

\section{Prognostic factors influencing the survival}

The correlations between the progression-free survival and the clinicopathological factors are shown in Table 3. According to the results of a univariate analysis, the progression-free survival showed significant relationships with the density of TAMs $(p=0.002)$, lymphatic involvement $(p=0.011)$, lymph node metastasis $(p=0.001)$, CEA $(p=0.014)$, and CA19-9 $(p<0.001)$. A multivariate analysis indicated that the density of TAMs (hazard ratio: 3.692 ; $95 \%$ confidence interval: 1.743-7.822; $p=0.001$ ) and lymph node metastasis (hazard ratio: 2.251; 95\% confidence interval: $1.131-4.481 ; p=0.021$ ) were independent prognostic factors for the progressionfree survival.

The correlations between the overall survival and the clinicopathological factors are shown in Table 4. According to the results of a univariate analysis, the overall survival showed significant relationships with the density

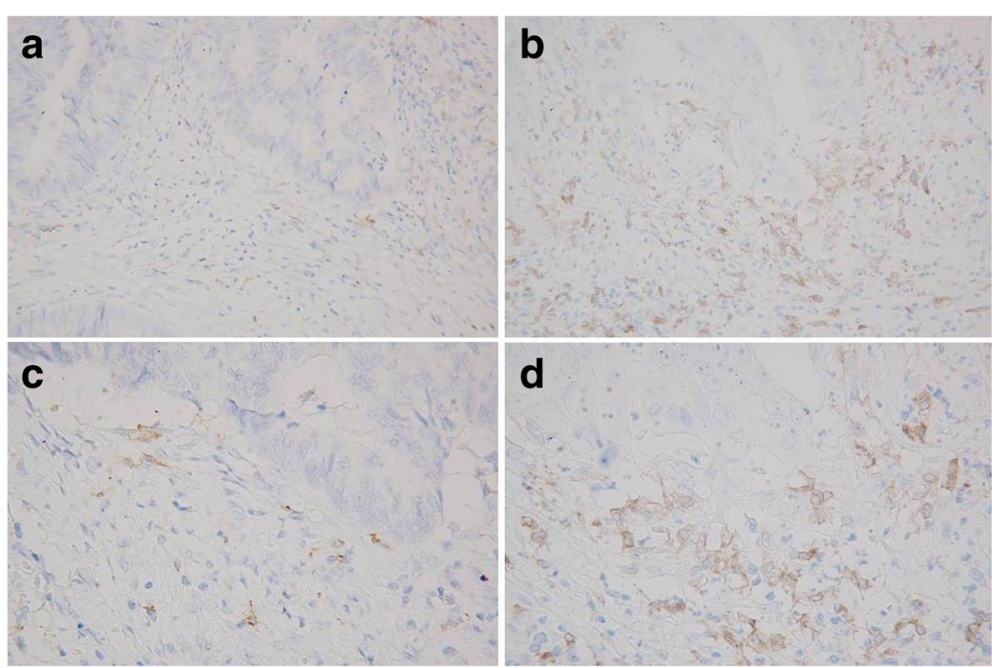

Fig. 1 The immunohistochemical expression of CD163, an M2 macrophage-specific marker. a A low density of TAMs (100x) b A high density of TAMs (100x) c A low density of TAMs (400x) d A high density of TAMs (400x) 
Table 2 Correlations between the density of TAMs and clinicopathological factors

\begin{tabular}{|c|c|c|c|}
\hline & \multicolumn{2}{|l|}{ TAM } & \multirow[b]{2}{*}{$p$-value } \\
\hline & Low & High & \\
\hline \multicolumn{4}{|l|}{ Age (years) } \\
\hline$<70$ & 50 & 59 & \\
\hline$\geq 70$ & 35 & 24 & 0.108 \\
\hline \multicolumn{4}{|l|}{ Gender } \\
\hline Male & 37 & 47 & \\
\hline Female & 48 & 35 & 0.089 \\
\hline \multicolumn{4}{|l|}{ Tumor depth ${ }^{a}$} \\
\hline $\mathrm{T} 1-3$ & 58 & 51 & \\
\hline T4 & 27 & 32 & 0.420 \\
\hline \multicolumn{4}{|l|}{ Histological type } \\
\hline Well, moderate & 82 & 72 & \\
\hline Poorly, mucinous & 3 & 11 & 0.027 \\
\hline \multicolumn{4}{|l|}{ Tumor diameter } \\
\hline$<5 \mathrm{~cm}$ & 58 & 49 & \\
\hline$\geq 5 \mathrm{~cm}$ & 27 & 34 & 0.262 \\
\hline \multicolumn{4}{|c|}{ Lymphatic involvement } \\
\hline Negative & 20 & 20 & \\
\hline Positive & 65 & 63 & 1.000 \\
\hline \multicolumn{4}{|l|}{ Venous involvement } \\
\hline Negative & 70 & 67 & \\
\hline Positive & 15 & 16 & 0.844 \\
\hline \multicolumn{4}{|c|}{ Lymph node metastasis } \\
\hline Negative & 46 & 46 & \\
\hline Positive & 39 & 37 & 0.878 \\
\hline \multicolumn{4}{|l|}{ CEA } \\
\hline$\leq 5 \mathrm{ng} / \mathrm{ml}$ & 56 & 49 & \\
\hline$>5 \mathrm{ng} / \mathrm{ml}$ & 29 & 34 & 0.426 \\
\hline \multicolumn{4}{|l|}{ CA19-9 } \\
\hline$\leq 37 \mathrm{U} / \mathrm{ml}$ & 76 & 71 & \\
\hline$>37 \mathrm{U} / \mathrm{ml}$ & 6 & 12 & 0.211 \\
\hline
\end{tabular}

TAMs tumor-associated macrophages, CEA carcinoembryonic antigen, CA19-9 carbohydrate antigen 19-9

${ }^{a}$ :According to the UICC. TNM Classification of Malignant Tumors (Seventh edition)

of TAMs $(p=0.027)$, age $(p=0.036)$, venous involvement $(p=0.010)$, lymph node metastasis $(p=0.024)$, CEA $(p=0.021)$, and CA19-9 $(p=0.017)$. A multivariate analysis indicated that the density of TAMs (hazard ratio: 4.123; 95\% confidence interval: 1.464-11.610; $p=0.007$ ), age (hazard ratio: $3.355 ; 95 \%$ confidence interval: $1.373-8.200 ; p=0.008)$, and venous involvement (hazard ratio: 3.911; 95\% confidence interval: 1.540-9.936; $p=0.004$ ) were independent prognostic factors for the overall survival.

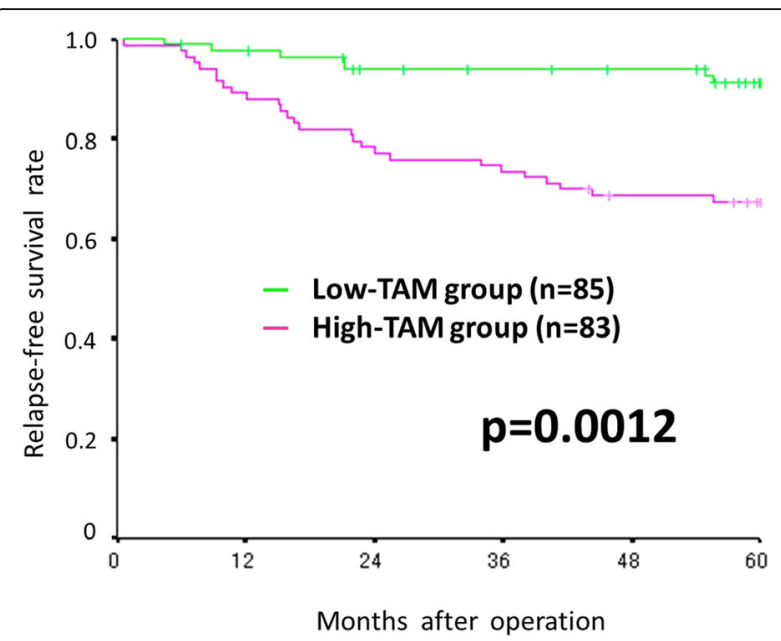

Fig. 2 Kaplan-Meier survival curves for the relapse-free survival according to the density of TAMs. The relapse-free survival rate was significantly worse in the high-TAMs group than in the low-TAMs group $(p=0.0012)$

Correlation between the peripheral monocyte count and the number of TAMs in the tumor microenvironment

The peripheral monocyte count was significantly associated with the number of TAMs (correlation coefficient: 0.202, $p=0.047$ ) (Fig. 4).

\section{Discussion}

We found that a high density of TAMs in the cancer microenvironment was associated with a poor prognosis in patients with colorectal cancer. We also found that the peripheral monocyte count was associated with the density of TAMs in the cancer microenvironment. These results may explain the reason why the peripheral

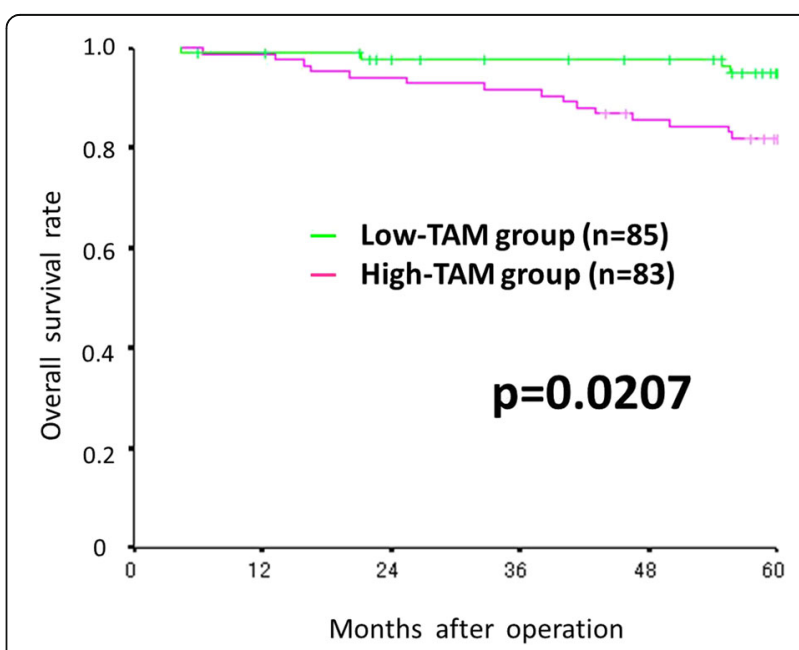

Fig. 3 Kaplan-Meier survival curves for the overall survival according to the density of TAMs. The overall survival rate was significantly worse in the high-TAMs group than in the low-TAMsgroup $(p=0.0207)$ 
Table 3 Correlations between the relapse-free survival and clinicopathological factors

\begin{tabular}{|c|c|c|c|c|c|c|}
\hline & \multicolumn{3}{|c|}{ Univariate analysis } & \multicolumn{3}{|c|}{ Multivariate analysis } \\
\hline & $\mathrm{HR}$ & $95 \% \mathrm{Cl}$ & $P$-value & $\mathrm{HR}$ & $95 \% \mathrm{Cl}$ & $P$-value \\
\hline Age ( $\geq 70$ years vs. $<70$ years) & 1.611 & $0.882-2.942$ & 0.121 & & & \\
\hline Gender (Male vs. Female) & 1.657 & $0.894-3.071$ & 0.109 & & & \\
\hline Tumor depth (T4 vs. T1-3) & 1.617 & $0.879-2.976$ & 0.123 & & & \\
\hline Histological type (Poorly, Mucinous vs. Well, Moderately) & 2.092 & $0.879-4.978$ & 0.095 & & & \\
\hline Lymphatic involvement (Positive vs. Negative) & 3.837 & $1.367-10.767$ & 0.011 & 2.563 & $0.879-7.471$ & 0.085 \\
\hline Venous involvement (Positive vs. Negative) & 1.857 & $0.953-3.621$ & 0.069 & & & \\
\hline Lymph node metastasis (Positive vs. Negative) & 3.016 & $1.589-5.724$ & 0.001 & 2.251 & $1.131-4.481$ & 0.021 \\
\hline CEA ( $>5 \mathrm{ng} / \mathrm{ml}$ vs. $\leq 5 \mathrm{ng} / \mathrm{ml}$ ) & 2.123 & $1.165-3.870$ & 0.014 & 1.332 & $0.667-2.660$ & 0.417 \\
\hline CA19-9 (>37 U/ml vs. $\leq 37 \mathrm{U} / \mathrm{ml})$ & 3.764 & $1.821-7.777$ & $<0.001$ & 1.821 & $0.782-4.242$ & 0.165 \\
\hline TAM (High vs. Low) & 2.973 & $1.493-5.920$ & 0.002 & 3.692 & $1.743-7.822$ & 0.001 \\
\hline
\end{tabular}

HR hazard ratio, Cl confidence interval, TAMs tumor-associated macrophages, CEA carcinoembryonic antigen, CA19-9 carbohydrate antigen 19-9

monocyte count functions as a prognostic marker in patients with colorectal cancer.

Increasing evidence suggests that stromal cells, such as tumor-infiltrating lymphocytes, TAMs, and cancerassociated fibroblasts, in the cancer microenvironment are associated with cancer progression. TAMs were first reported in the early 1980s [18] and have been extensively studied, with their process of differentiation and function now clear. Monocytes differentiate into macrophages after recruitment from the peripheral blood to the tumor [11, 12, 19]. They can be divided into two main phenotypes: M1 type and M2 type. These polarizations are adjusted by cytokines, such as macrophagecolony-stimulating factor (M-CSF), transforming growth factor (TGF)- $\beta$, interleukin (IL)- 6 and IL-10 in the cancer microenvironment $[20,21]$. M1 macrophages have antitumor activity, whereas M2 macrophages play an important role in invasion, metastasis, angiogenesis, and immunosuppression, which lead to cancer progression $[15,22]$. M2-macrophages play an important role in tumor progression and metastasis via angiogenesis through their production of angiogenic factors such as vascular endothelial growth factor (VEGF) [23, 24], and play a role in tumor invasion via a matrix metalloproteinase (MMP)-dependent mechanism through their production of tumor necrosis factor-alpha (TNF- $\alpha$ ) [25]. Moreover, M2 macrophages are responsible for immunosuppression through their inhibition of the $\mathrm{T}$ cell function via the programmed cell death-1 (PD-1)/programmed cell death-ligand 1 (PDL1) pathway and their production of immunosuppressive cytokines such as interleukin (IL)-10 [26]. Because the majority of TAMs have an M2-like phenotype [27], the high density of TAMs in the cancer microenvironment is associated with a poor prognosis.

In previous reports, the peripheral monocyte count and the lymphocyte-to-monocyte ratio have been reported to be useful prognostic markers $[9,10,16,28,29]$. This was based on the hypothesis that the peripheral monocyte count was associated with the density of TAMs in the cancer microenvironment. However, few reports have

Table 4 Correlations between the overall survival and clinicopathological factors

\begin{tabular}{|c|c|c|c|c|c|c|}
\hline & \multicolumn{3}{|c|}{ Univariate analysis } & \multicolumn{3}{|c|}{ Multivariate analysis } \\
\hline & $\mathrm{HR}$ & $95 \% \mathrm{Cl}$ & $P$-value & $\mathrm{HR}$ & $95 \% \mathrm{Cl}$ & $P$-value \\
\hline Age ( $\geq 70$ years vs. $<70$ years) & 2.366 & $1.058-5.289$ & 0.036 & 3.355 & $1.373-8.200$ & 0.008 \\
\hline Gender (Male vs. Female) & 1.250 & $0.565-2.765$ & 0.582 & & & \\
\hline Tumor depth (T4 vs. T1-3) & 1.387 & $0.616-3.124$ & 0.430 & & & \\
\hline Histological type (Poorly, Mucinous vs. Well, Moderately) & 1.660 & $0.495-5.573$ & 0.412 & & & \\
\hline Lymphatic involvement (Positive vs. Negative) & 2.461 & $0.733-8.258$ & 0.145 & & & \\
\hline Venous involvement (Positive vs. Negative) & 2.987 & $1.306-6.831$ & 0.010 & 3.911 & $1.540-9.936$ & 0.004 \\
\hline Lymph node metastasis (Positive vs. Negative) & 2.641 & $1.135-6.148$ & 0.024 & 1.729 & $0.699-4.273$ & 0.236 \\
\hline CEA (>5 ng/ml vs. $\leq 5 \mathrm{ng} / \mathrm{ml}$ ) & 2.563 & $1.149-5.717$ & 0.021 & 1.258 & $0.513-3.085$ & 0.616 \\
\hline CA19-9 (>37 U/ml vs. $\leq 37 \mathrm{U} / \mathrm{ml})$ & 3.117 & $1.226-7.928$ & 0.017 & 2.127 & $0.720-6.281$ & 0.172 \\
\hline TAM (High vs. Low) & 2.841 & $1.128-7.152$ & 0.027 & 4.123 & $1.464-11.610$ & 0.007 \\
\hline
\end{tabular}

HR hazard ratio, Cl confidence interval, TAMs tumor-associated macrophages, CEA carcinoembryonic antigen, CA19-9 carbohydrate antigen 19-9 


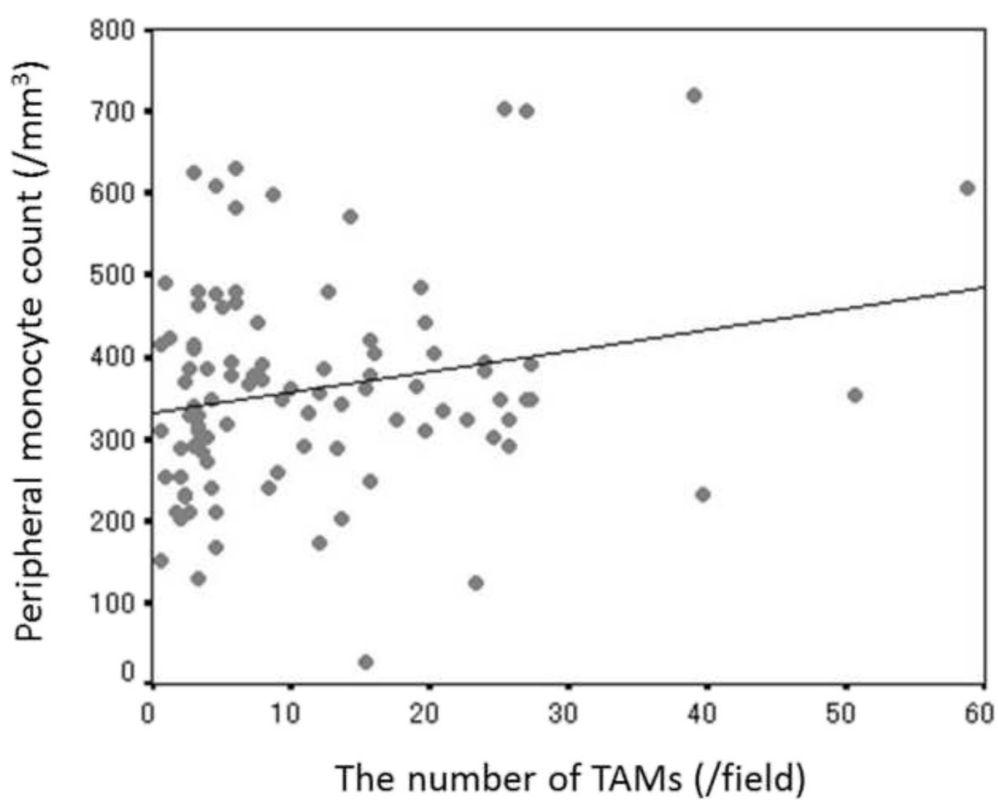

Fig. 4 The correlation between the peripheral monocyte count and the number of TAMs in the tumor microenvironment (correlation coefficient: $0.202, p=0.047$ )

described the correlation between the peripheral monocyte count and the density of TAMs in the cancer microenvironment. In the present study, the peripheral monocyte count was shown to correlate with the density of TAMs in the cancer microenvironment, suggesting that inflammatory markers such as the peripheral monocyte count might be surrogate markers reflecting the status of the cancer microenvironment. A peripheral blood cell count is a quick, easy, and inexpensive assay to perform and is often carried out as a routine examination. We hope that peripheral inflammatory markers will be applied clinically as biomarkers in patients with colorectal cancer in the future.

The median peripheral monocyte count, which was obtained 5 years after operation from patients who had been relapse free, was 321 (range: 118-504). This value was significantly lower than the preoperative peripheral monocyte count $(p<0.001$, paired $t$-test). The mechanism underlying the increase in the peripheral monocyte count of cancer patients is considered to be as follows. Chemokines (such as CCL2), which are produced by cancer cells, promote the recruitment of peripheral monocytes to the cancer microenvironment, thereby promoting the recruitment of monocytes from the bone marrow to peripheral blood.

Several limitations associated with the present study warrant mention. First, we evaluated a relatively small number of patients, and the study design was retrospective. Second, factors other than the response of the host to the cancer, which affect the systemic inflammation, were not assessed. Third, M1 macrophages, which are also derived from circulating monocytes, were not considered in this study, although most macrophages in the cancer microenvironment are reported to be M2 macrophages, and the impact of M1 macrophages on the cancer microenvironment is likely negligible. Fourth, we did not verify the polarization of the macrophages in this study. Further studies are needed to elucidate the mechanisms underlying M1/M2 polarization in the cancer microenvironment. By co-culturing the peripheral monocytes and cancer cell lines, we can confirm that most monocytes polarized to the M2 phenotype in the cancer microenvironment and investigate the types of cytokines that are involved in polarization.

\section{Conclusions}

In conclusion, our results showed that the peripheral monocyte count was associated with the density of the TAMs, which created a microenvironment favorable for cancer development and were correlated with a poor prognosis, in the cancer microenvironment. Therefore, the peripheral monocyte count is considered to be a useful prognostic marker reflecting the status of the tumor microenvironment.

\section{Abbreviations}

CA19-9: Carbohydrate antigen 19-9; CEA: Carcinoembryonic antigen: Cl: Confidence interval; HR: Hazard ratio; IL: Interleukin; M-CSF: Macrophagecolony-stimulating factor; TAMs: Tumor-associated macrophages; TGF- $\beta$ : Transforming growth factor- $\beta$ 


\section{Acknowledgements}

This research received no specific grants from any funding agency in the public, commercial or not-for-profit sectors. We thank Brian Quinn who provided medical writing services on behalf of JMC, Ltd.

\section{Funding}

No funding was acquired for this study.

\section{Availability of data and materials}

The datasets used and/or analyzed during the current study are available from the corresponding author on reasonable request.

\section{Authors' contributions}

MS and KM designed the study, performed the statistical analysis and draft the manuscript. HN, TF, SN and SM collected the clinical data and revised the manuscript critically. $\mathrm{KH}$ and $\mathrm{MO}$ designed the study and critically reviewed the manuscript. All authors read and approved the final manuscript.

\section{Competing interests}

The authors declare that they have no competing interests.

\section{Consent for publication}

Not applicable.

\section{Ethics approval and consent to participate}

This research conformed to the provisions of the Declaration of Helsinki. All patients were informed of the investigational nature of this study and provided their written informed consent. This retrospective study was approved by the ethics committee of Osaka City University (approved No.926).

\section{Publisher's Note}

Springer Nature remains neutral with regard to jurisdictional claims in published maps and institutional affiliations.

Received: 17 March 2017 Accepted: 30 May 2017 Published online: 05 June 2017

\section{References}

1. Mantovani A, Allavena P, Sica A, Balkwill F. Cancer-related inflammation. Nature. 2008;454:436-44.

2. Baba H, Kuwabara K, Ishiguro T, Hatano S, Matsuzawa T, Fukuchi M, et al. Creactive protein as a significant prognostic factor for stage IV gastric cancer patients. Anticancer Res. 2013:33:5591-5.

3. Shibutani M, Maeda K, Nagahara H, Noda E, Ohtani H, Nishiguchi Y, et al. A high preoperative neutrophil-to-lymphocyte ratio is associated with poor survival in patients with colorectal cancer. Anticancer Res. 2013;33:3291-4.

4. Luo G, Guo M, Liu Z, Xiao Z, Jin K, Long J, et al. Blood neutrophillymphocyte ratio predicts survival in patients with advanced pancreatic cancer treated with chemotherapy. Ann Surg Oncol. 2015;22:670-6.

5. Fan H, Shao ZY, Xiao YY, Xie ZH, Chen W, Xie H, et al. Comparison of the Glasgow Prognostic Score (GPS) and the modified Glasgow Prognostic Score (mGPS) in evaluating the prognosis of patients with operable and inoperable non-small cell lung cancer. J Cancer Res Clin Oncol. 2016;142: 1285-97.

6. Shibutani M, Maeda K, Nagahara H, Iseki Y, Ikeya T, Hirakawa K. Prognostic significance of the preoperative ratio of $\mathrm{C}$-reactive protein to albumin in patients with colorectal cancer. Anticancer Res. 2016;36:995-1001.

7. Shigeta K, Kosaka T, Kitano S, Yasumizu Y, Miyazaki Y, Mizuno R, et al. High absolute monocyte count predicts poor clinical outcome in patients with castration-resistant prostate cancer treated with docetaxel chemotherapy. Ann Surg Oncol. 2016;23:4115-22.

8. He J, LV P, Yang X, Chen Y, Liu C, Qiu X. Pretreatment lymphocyte to monocyte ratio as a predictor of prognosis in patients with early-stage triple-negative breast cancer. Tumour Biol. 2016;37:9037-43.

9. Chan JC, Chan DL, Diakos Cl, Engel A, Pavlakis N, Gill A, et al. The Lymphocyte-to-monocyte ratio is a superior predictor of overall survival in comparison to established biomarkers of resectable colorectal cancer. Ann Surg. 2017;265:539-46.
10. Shibutani M, Maeda K, Nagahara H, Iseki Y, Ikeya T, Hirakawa K. Prognostic significance of the preoperative lymphocyte-to-monocyte ratio in patients with colorectal cancer. Oncol Lett. 2017;13:1000-6.

11. Gosselin D, Link VM, Romanoski CE, Fonseca GJ, Eichenfield DZ, Spann NJ, et al. Environment drives selection and function of enhancers controlling tissue-specific macrophage identities. Cell. 2014;159:1327-40.

12. Marech I, Ammendola M, Sacco R, Sammarco G, Zuccalà V, Zizzo N, et al. Tumour-associated macrophages correlate with microvascular bed extension in colorectal cancer patients. J Cell Mol Med. 2016;20: 1373-80.

13. Maniecki MB, Etzerodt A, Ulhøi BP, Steiniche T, Borre M, Dyrskjøt L, et al. Tumor-promoting macrophages induce the expression of the macrophage-specific receptor CD163 in malignant cells. Int J Cancer. 2012;131:2320-31.

14. Richards DM, Hettinger J, Feuerer M. Monocytes and macrophages in cancer: development and functions. Cancer Microenviron. 2013;6:179-91.

15. De Palma M, Lewis CE. Macrophage regulation of tumor responses to anticancer therapies. Cancer Cell. 2013;23:277-86.

16. Shibutani M, Maeda K, Nagahara H, Ohtani H, Sakurai K, Yamazoe S, et al. Prognostic significance of the lymphocyte-to-monocyte ratio in patients with metastatic colorectal cancer. World J Gastroenterol. 2015;21:9966-73.

17. Sobin LH, Gospodarowicz MK, Wittekind C. UICC. TNM Classification of Malignant Tumors. Seventh edition. New York: Wiley-Liss; 2009.

18. Badawi MA, Abouelfadl DM, El-Sharkawy SL, El-Aal WE, Abbas NF. Tumor-Associated Macrophage (TAM) and angiogenesis in human colon carcinoma. Open Access Maced J Med Sci. 2015;3:209-14.

19. Mantovani A, Bottazzi B, Colotta F, Sozzani S, Ruco L. The origin and function of tumor-associated macrophages. Immunol Today. 1992;13:265-70.

20. Zhang F, Wang $H$, Wang $X$, Jiang G, Liu H, Zhang G, et al. TGF- $\beta$ induces M2-like macrophage polarization via SNAIL-mediated suppression of a proinflammatory phenotype. Oncotarget. 2016;7:52294-306.

21. Sica A, Schioppa T, Mantovani A, Allavena P. Tumour-associated macrophages are a distinct $\mathrm{M} 2$ polarised population promoting tumour progression: potential targets of anti-cancer therapy. Eur J Cancer. 2006;42:717-27.

22. Mantovani A, Sozzani S, Locati M, Allavena P, Sica A. Macrophage polarization: tumor-associated macrophages as a paradigm for polarized M2 mononuclear phagocytes. Trends Immunol. 2002;23:549-55.

23. Kimura YN, Watari K, Fotovati A, Hosoi F, Yasumoto K, Izumi H, et al. Inflammatory stimuli from macrophages and cancer cells synergistically promote tumor growth and angiogenesis. Cancer Sci. 2007;98:2009-18.

24. Carmeliet $P$, Jain RK. Angiogenesis in cancer and other diseases. Nature. 2000:407:249-57.

25. Hagemann T, Robinson SC, Schulz M, Trümper L, Balkwill FR, Binder C. Enhanced invasiveness of breast cancer cell lines upon co-cultivation with macrophages is due to TNF-alpha dependent up-regulation of matrix metalloproteases. Carcinogenesis. 2004;25:1543-9.

26. Mantovani A, Allavena P. The interaction of anticancer therapies with tumor-associated macrophages. J Exp Med. 2015:212:435-45.

27. He KF, Zhang L, Huang CF, Ma SR, Wang YF, Wang WM, et al. CD163+ tumor-associated macrophages correlated with poor prognosis and cancer stem cells in oral squamous cell carcinoma. Biomed Res Int. 2014;2014:838632.

28. Stotz M, Pichler M, Absenger G, Szkandera J, Arminger F, Schaberl-Moser R, et al. The preoperative lymphocyte to monocyte ratio predicts clinical outcome in patients with stage III colon cancer. Br J Cancer. 2014;110:435-40.

29. Hu P, Shen $H$, Wang G, Zhang $P$, Liu Q, Du J. Prognostic significance of systemic inflammation-based lymphocyte- monocyte ratio in patients with lung cancer: based on a large cohort study. PLoS One. 2014;9:e108062 